\title{
Multi-View Fusion Network for Crop Disease Recognition
}

\author{
Lihong Xie \\ Ruiling Han \\ Songhong Xie \\ Amazingx Academy, Shenzhen, China Amazingx Academy, Shenzhen, China Amazingx Academy, Shenzhen, China \\ Dongjing Chen \\ Amazingx Academy, Shenzhen, China \\ Yaxuan $\mathrm{CHEN}^{*}$ \\ School of Computer Science, Wuhan \\ Donghu University, Wuhan, China
}

\begin{abstract}
During the growth of crops, crop yields will be affected by various diseases. Automatic and accurate recognition of crop diseases and determination of disease severity are the key to crop disease prevention and control. In practice, due to noise interference in the data collection process, etc., the acquired crop disease recognition datasets are often with large intra-class differences and large inter-class similarities, which brings great challenges to the development of accurate crop disease recognition. Existing crop disease recognition methods often ignore the subtle differences in easily confused categories, which leads to limited performance. To this end, this paper proposes a multi-view fusion network (MVF-Net) for crop disease recognition. The proposed MVF-Net consists of two parallel branches: suppression branch and global branch. The suppression branch explores discriminative fine-grained features by suppressing the saliency information of the image. The global branch aims to learn the global features of the image to avoid the network paying too much attention to local features. In addition, in order to promote network learning with more discriminative features, this paper introduces an embedding loss, which is combined with the traditional multi-class cross-entropy loss function to improve the performance of the crop disease recognition model. Extensive experiments are conducted on the 2018ai_challenger crop disease recognition dataset. The results show that the proposed MVF-Net has certain advantages over other comparison methods, which lays the foundation for the prevention and control of crop diseases.
\end{abstract}

\section{CCS CONCEPTS}

- Computing methodologies; • Modeling methodologies;

\section{KEYWORDS}

Crop disease recognition, Multi-view fusion, Fine-grained features, Global features, Attention

ACM Reference Format:

Lihong Xie, Ruiling Han, Songhong Xie, Dongjing Chen, and Yaxuan CHEN* 2021. Multi-View Fusion Network for Crop Disease Recognition. In 2021 The 5th International Conference on Algorithms, Computing and Systems (ICACS

Permission to make digital or hard copies of all or part of this work for personal or classroom use is granted without fee provided that copies are not made or distributed for profit or commercial advantage and that copies bear this notice and the full citation on the first page. Copyrights for components of this work owned by others than ACM must be honored. Abstracting with credit is permitted. To copy otherwise, or republish, to post on servers or to redistribute to lists, requires prior specific permission and/or a fee. Request permissions from permissions@acm.org.

ICACS '21, September 24-26, 2021, Xi'an, China

(C) 2021 Association for Computing Machinery.

ACM ISBN 978-1-4503-8508-4/21/09 . \$ \$15.00

https://doi.org/10.1145/3490700.3490724
'21), September 24-26, 2021, Xi'an, China. ACM, New York, NY, USA, 6 pages. https://doi.org/10.1145/3490700.3490724

\section{INTRODUCTION}

Crop disease is one of the main agricultural disasters in the world. It has the characteristics of many types, great impact and frequent outbreaks. It not only causes losses to crop production, but also threatens food safety [1]. The correct diagnosis and recognition of crop diseases plays an important role in ensuring crop yields and preventing food safety. Identifying diseases requires professionals to conduct recognition, analysis, and draw conclusions. However, most planters have little professional knowledge and lack of experience. Therefore, it is urgent to use modern computer and Internet technology to realize that farmers can judge crop diseases by shooting images of diseased leaves and make timely prevention of diseases [2].

\subsection{Related Work and Motivation}

With the continuous development of deep learning (DL) and image technology, the use of high-performance parallel computing clusters has greatly increased the calculation speed, making the Convolutional Neural Network (CNN) has made great progress in target detection [3-5] and image classification [6, 7]. It makes up for the disadvantages of poor feature extraction capabilities in traditional image classification methods, and the inability to perform classification based on the acquired feature expressions after complex image preprocessing and classification algorithms. The use of CNN combined with computer vision technology to identify agricultural diseases has become a current research hotspot. Kawasaki et al. [8] used a convolutional neural network to propose a cucumber leaf disease diagnosis system, which achieved $94.9 \%$ accuracy in terms of melon yellow spot virus, zucchini yellow mosaic virus and non-disease categories. Sanyal et al. [9] diagnosed rice diseases such as brown spot and rice blast based on the color and texture of rice leaves. Mohit et al. [10] proposed a light weight model for tomato crop disease recognition, which is better than traditional machine learning (ML) methods. Srdjan et al. [11] used convolutional neural networks to realize the recognition of 13 diseases. The model also has the ability to distinguish between plant leaves and their surrounding environment, and finally obtains a higher recognition rate. Mohanty et al. utilized AlexNet and GoogLeNet model training to classify and recognize images of 14 plants, 59 diseases, and some healthy plants in PlantVillage. The recognition accuracy can reach $97.82 \%$ and $99.35 \%$, respectively. Brahimi et al. [13] collected 14,828 images to identify 59 types of diseases on tomato leaves, with an accuracy rate of $99.18 \%$. Jihen et al. [14] applied LeNet network to classify banana diseases and achieved a high recognition rate. 


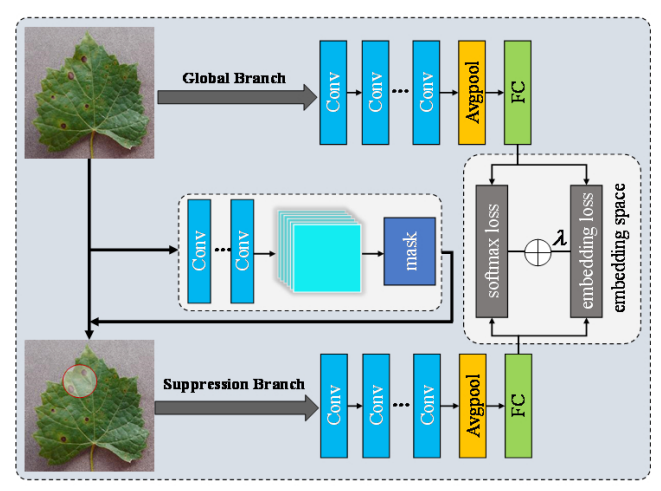

Figure 1: The framework of the proposed MVF-Net.

In order to improve the robustness and accuracy of recognition, Zeng et al. proposed a self-attention convolutional neural network to identify crop diseases by extracting effective features of crop disease spots. Nagasubramanian et al. [15] proposed a crop monitoring system that integrates support vector machines (SVM) and $\mathrm{CNN}$ for crop growth and leaf disease monitoring.

These methods have greatly promoted the development of automatic recognition of crop diseases, but they tend to pay too much attention to the salient areas in the image, and ignore other small clues that contain complementary information that are conducive to differentiation. This makes it difficult for the existing methods to achieve satisfactory performance.

\subsection{Overview}

In order to solve the problem, this paper proposes a multi-view fusion network (MVF-Net) for crop disease recognition that combines global and local features. The proposed MVF-Net forces the network to learn the subtle features of easily confused categories by suppressing the most prominent areas in the crop leaf images, thereby accurately distinguishing different types of diseases. In order to avoid the network paying too much attention to local features, this paper also introduces a global branch to learn the overall structural features of the image. Considering that the traditional multi-class cross-entropy loss function can increase the distance between different classes, the data is separable between classes in the feature space. But for crop disease recognition, compactness within the class is also very important. Therefore, on this basis, this paper also proposes a new embedding loss, by reducing the distance between each image and its corresponding class center. By fully mining the correlation between features, the learned features more discriminative.

\subsection{Contributions}

In summary, the contributions of this paper include the following 3 points:

1. A multi-view fusion network (MVF-Net) for crop disease recognition is proposed, which can effectively integrate global and local features and improve the performance of crop disease recognition models.

2. A feature suppression branch is designed to force the network to learn subtle features between categories that are easy to confuse by suppressing the most prominent areas in the crop leaf images, thereby accurately distinguishing different types of diseases.

3. A new loss function, embedding loss, is introduced, which is combined with the traditional multi-class cross-entropy loss function to make each type of data more compact in the feature space, thereby improving the performance of the crop disease recognition model.

\subsection{Organization}

The remaining sections are organized as follows: Section 2 dwells on the proposed method. Section 3 reports the experimental setup and results, meanwhile gives a discussion. Section 4 concludes the paper.

\section{THE PROPOSED METHOD}

The framework of the proposed in MVF-Net is shown in Figure 1, including two branches: global branch and suppression branch. Specifically, the global branch directly inputs fixed-size original crop images into the classification network for training. The suppression branch generates a suppression image with a local area masked based on the feature map. Then, it is input to the classification network for training, thereby distracting attention and making the network focus on complementary areas that contain subtle clues. In addition, an embedding space is introduced, in which a novel embedding loss function is designed. The loss function is combined with the softmax loss function to increase the distance between samples of different types and reduce the distance between samples of the same type, making the resulting features more discriminative.

\subsection{Bilinear Pooling}

After extracting image features through the convolutional layer, inspired by the bilinear pooling model proposed by Lin et al. [16], 


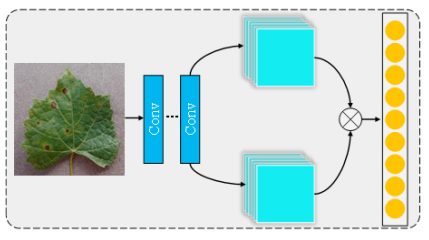

Figure 2: The architecture of the bilinear pooling.

the features are fused to make the acquired features more discriminative. Moreover, the bilinear pooling has a simple form, which facilitates the back propagation of gradients, thereby realizing endto-end training.

Specifically, as shown in Figure 2, the feature maps output by conv1 and conv2 from the second block of layer4 in ResNet-50 [17] are denoted as $\mathbf{F}_{1} \in \mathbb{R}^{C_{1} \times H \times W}$ and $\mathbf{F}_{2} \in \mathbb{R}^{C_{2} \times H \times W}$, respectively, where $C_{1}$ and $C_{2}$ represent the number of channels of the two features. $H$ and $W$ represent the height and width of the feature map, respectively. In order to solve the problem of too high feature dimension after fusion, while ensuring that the feature information contained in the generated feature vector is sufficient, only the feature maps of $n$ channels in $F_{2}$ are randomly selected for fusion with $\mathbf{F}_{1}$. Note that the feature vectors along the channel at each position in the feature maps $\mathbf{F}_{1}$ and $\mathbf{F}_{2}$ are $\mathbf{V}_{1} \in \mathbb{R}^{C_{1} \times 1}$ and $\mathbf{V}_{2} \in$ $\mathbb{R}^{C_{2} \times 1}$. After the two eigenvectors are multiplied according to Eq. 1 , the bilinear matrix $\mathbf{B} \in \mathbb{R}^{C_{1} \times C_{2}}$ can be obtained.

$$
\mathbf{B}=\mathbf{V}_{1} \mathbf{V}_{2}^{T} \text {, }
$$

The bilinear matrix corresponding to each position in the feature map $\mathbf{B}$ is added, and the matrix is expanded into a vector, which is a bilinear vector. The bilinear vector contains rich feature information, which can be input into the embedding space proposed in Section 2.3 to be constrained.

\subsection{Suppression Branch}

In previous works $[18,19]$, convolutional neural networks based on the cross-entropy loss function often focus on some salient areas, while ignoring other tiny clues that contain complementary information. Since there are often some very subtle differences between the easily confused categories in crop disease recognition, it is necessary to pay attention to subtle features. In this study, by suppressing the salient areas in the image, the attention is distracted, so as to capture the relationship between various subtle features in the image.

Specifically, the feature map $\mathbf{F}_{2} \in \mathbb{R}^{C_{2} \times H \times W}$ randomly selected in section 2.1 is average pooled and sorted in descending order. The first feature map after sorting is selected, and enlarged to the original image size. Then, the average value $A$ of the feature map is calculated. Using $\theta * A$ as the threshold, the elements larger than the threshold in the feature map are set to 0 , and other elements are set to 1 , so as to obtain the suppression mask $\mathbf{M}_{k}$ :

$$
\mathbf{M}_{k}(i, j)=\left\{\begin{array}{c}
0, \text { if } \mathbf{M}(i, j)>\theta * A \\
1, \text { otherwise }
\end{array},\right.
$$

The suppression mask is covered to the original image to suppress the $k$-th component area, thereby forcing the neural network to learn discriminative information from other areas. The above operations can effectively suppress the most significant areas of the image, distract attention. In addition, it can also reduce the network's dependence on training samples, prevent overfitting, and further improve the robustness of the model.

\subsection{Embedding Space}

In order to make the extracted features more discriminative, the image is mapped into an embedding space. In the space, the distance of the images under the same category is shortened, and the distance of the images under different categories is shortened. In previous work, Schroff et al. applied triplet loss as the embedding loss [20]. The loss function randomly selects a sample from the training dataset, which is called an anchor. Then, a positive sample that belongs to the same class as the anchor and a negative sample that belongs to different class with the anchor are randomly selected, so as to form a triple. The purpose of triplet loss is to make the distance of feature representations from the same class as small as possible, while the distance of feature representations from different class as large as possible. However, the application of triplet loss requires the construction of a large number of training triples. In order to speed up the network convergence, this paper adopts a novel loss function, namely embedding loss, to explore the embedding space. The embedding loss $\ell_{\boldsymbol{e}}$ consists of the following two terms:

$$
\ell_{e}=\ell_{w}+\ell_{b},
$$

where the $\ell_{w}$ loss function minimizes the distance between each image and the center of its corresponding class, and the $\ell_{b}$ loss function maximizes the distance between the centers of different classes.

The $\ell_{w}$ loss function is derived from the central loss proposed by Wen et al. [21]. For each category, a feature vector is calculated as the center of the corresponding category. This feature vector will be updated continuously as the training progresses. By penalizing the deviation between the bilinear feature vector of each sample and the center of the corresponding class of samples, the samples from the same class are grouped together as much as possible. This saves the complicated training triples construction process. Let $x_{i}$ denote the bilinear feature of the $i$-th sample, and $c_{y i}$ denote the average feature of all samples in the class corresponding to the $i$-thsample, that is, the class center. The form of the $\ell_{w}$ loss function is as follows:

$$
\ell_{w}=\frac{1}{N} \sum_{i=1}^{N}\left\|x_{i}-c_{y i}\right\|_{2}^{2},
$$

where $N$ represents the number of samples in the current batch.

The $\ell_{b}$ loss function maximizes the distance between the centers of the updated classes, so that the distance between the feature expressions of samples from different categories are made as large as possible. $m$ defines the threshold of penalty distance, and $P$ represents the number of current batch categories. The $\ell_{b}$ loss function 


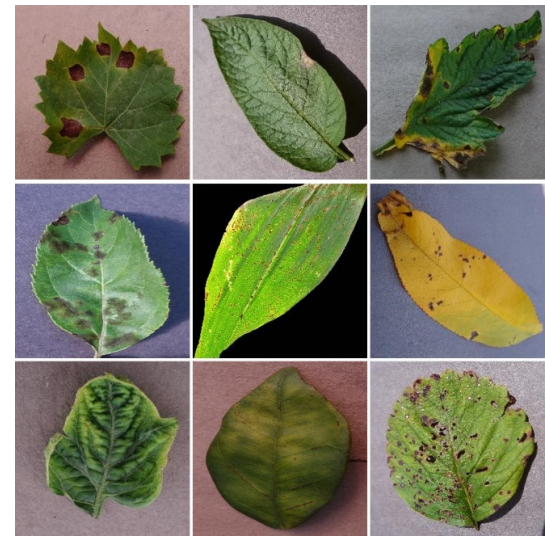

Figure 3: Some examples of the dataset.

can be expressed in the following form:

$$
\ell_{b}=\frac{1}{P} \sum_{i, j} \max \left(m-\left\|c_{y i}-c_{y j}\right\|_{2}^{3}, 0\right)^{2}
$$

The final loss function is a combination of softmax loss $\ell_{s}$ and embedding loss $\ell_{e}$ as following:

$$
\ell_{\text {final }}=\ell_{s}+\eta \ell_{e},
$$

where $\eta$ represents the trade-off coefficient between softmax loss and embedding loss, which is set to 0.8 in this work.

\section{EXPERIMENTS}

\subsection{Experimental Setup}

3.1.1 Dataset. The image data in this study comes from the plant disease dataset in the 2018ai_challenger competition. The dataset contains 35861 images of diseased leaves with different sizes. Since the $\mathrm{CNN}$ requires the size of the input images to be uniform, the image filling method is used to fill the image and then the size is scaled to an image with the size of $224 \times 224$ pixels. The dataset contains 10 kinds of crops. Because the symptoms of some diseases are quite different in the early and later stages, these diseases of different degrees are divided into different categories, a total of 59 categories. Some examples of the dataset are shown in Figure 3. In the experiment, we divided the dataset into training set, validation set and test set according to the ratio of 8:1:1.

3.1.2 Implementation Details. All experimental models are trained for 100 epochs. The SGD is employed as optimizer. The initial learning rate is set to 0.001 . Every two training epochs, the learning rate drops 0.9 of the original. The weight attenuation coefficient and the momentum coefficient are set to 0.00001 and 0.9 , respectively. The compromise factor $\eta$ in Eq. 6 is set to 0.8 .

In the training of the crop disease recognition model, the experimental hardware environment adopts two GeForce GTX 2080Ti GPUs. The software environment is the Ubuntu16.04 operating system and the Tensorflow deep learning framework. The Keras version is 2.2.4, the version of CUDA API is 9.1, and the version of cuDNN is 9.0. The algorithm implementation language adopts Python 3.6.3 version.

\subsection{Model Convergence}

In order to verify the convergence of the proposed MVF-Net, we recorded the loss and accuracy changes of the model during the training process. Figure 4(a) shows the loss curves of the training set and the validation set during the training of the crop disease model. Figure 4(b) shows the accuracy curves on the training set and the validation set. On the whole, the loss value during the model training process shows a downward trend, and the accuracy value shows an upward trend. After rapid convergence, the value shows a trend of oscillating in a smaller range. Comparing the curve change trends of the training set and the validation set, it can be seen that the change trends of the loss value and the accuracy value between the two are basically the same. This indicate that the overall network convergence is good, there is no over-fitting situation, and the convergence speed is faster. The model tends to be stable between 60 and 100 epochs.

\subsection{Ablation Study}

The model in this paper is mainly composed of three parts, including global branch, suppression branch, and embedding space. In order to verify the effectiveness of each component in the model, ResNet50 is used as the backbone network to conduct ablation experiments. In other words, we remove some components to study its impact on network performance in order to better understand the behavior of the network. Table 1 lists the overall recognition accuracy of the model and the recognition accuracy of individual components.

It can be seen from the results in Table 1 that when there is only a global branch, the recognition accuracy is $89.04 \%$. When the suppression branch is added to the global branch, the recognition accuracy is increased by $1.5 \%$. When the embedding space is added to the global branch, the accuracy is improved by nearly $0.6 \%$. The recognition accuracy of the proposed MVF-Net is $92.27 \%$, which is $3.2 \%$ higher than the global branch. It can be seen that the suppression branch diversifies the information area by forcing the network to find discriminative features other than the most prominent features. The embedding space introduces an embedding loss to make the extracted features more discriminative. Each component of the model contributes to performance.

\subsection{Comparison with Other Methods}

In order to verify the superiority of the proposed MVF-Net, we have selected several classification methods for comparison. The comparison methods include ResNet18 [17], ResNet50 [17], AlexNet [22], VGG16 [23] and SqueezeNet [24]. The number of neurons in the final classification layer is set as 59. It can be seen from Table 2 that the proposed MVF-Net model has the best performance while ensuring a smaller model size. Specifically, the recognition accuracy of the proposed MVF-Net is $92.27 \%$. Compared with ResNet18, ResNet50, AlexNet, VGG16 and SqueezeNet, the recognition accuracy is increased by $2.63 \%, 2.09 \%, 5.22 \%, 3.55 \%$ and $2.86 \%$, respectively. This mainly contains three reasons: 1 ) the proposed MVF-Net can force the network to learn the subtle features of easily confused categories by suppressing the most prominent areas in the crop leaf images, thereby accurately distinguishing different types of diseases; 2) the recognition process of the proposed MVF-Net comprehensively utilizes global and local features, making the recognition basis more 


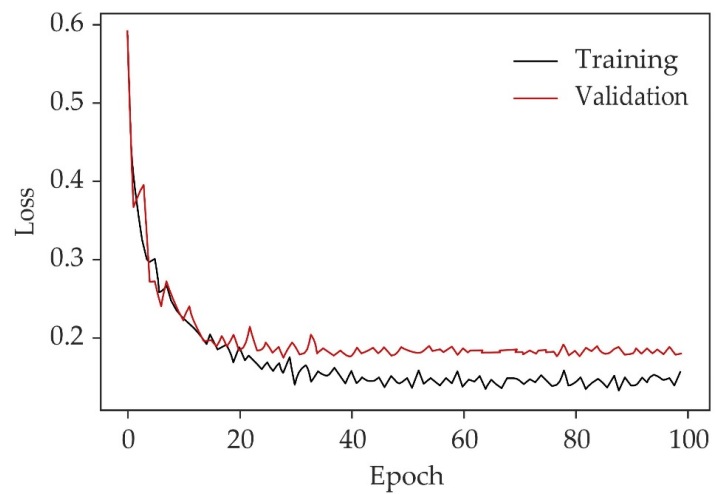

(a) Loss curve

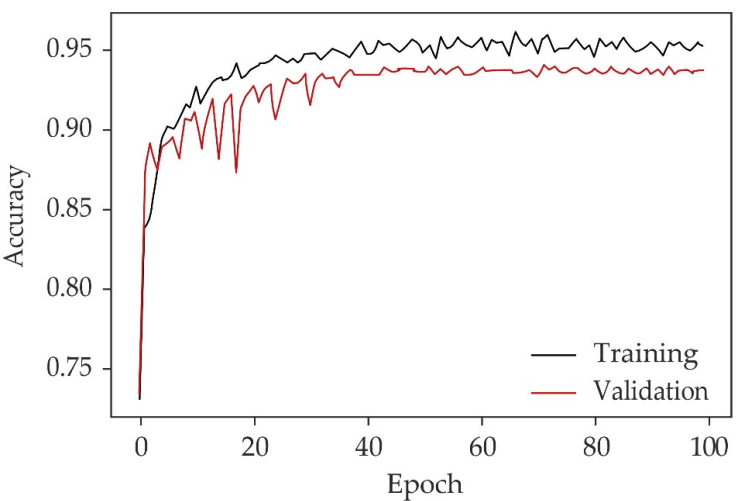

(b) Accuracy curve

Figure 4: The training and validation curves by the proposed MVF-Net.

Table 1: The ablation study for the proposed MVF-Net.

\begin{tabular}{llll}
\hline Global branch & Suppression branch & Embedding space & Accuracy $(\%)$ \\
\hline$\sqrt{ }$ & & & 89.04 \\
$\sqrt{ }$ & $\sqrt{ }$ & & 90.52 \\
$\sqrt{ }$ & $\sqrt{ }$ & $\sqrt{ }$ & 89.61 \\
$\sqrt{ }$ & $\sqrt{ }$ & 92.27 \\
\hline
\end{tabular}

Table 2: The comparison results with other methods.

\begin{tabular}{llll}
\hline Methods & Parameters & Model size $(\mathrm{Mb})$ & Accuracy $(\%)$ \\
\hline ResNet18 [17] & 11184207 & 106.02 & 89.64 \\
ResNet50 [17] & 23538767 & 376.92 & 90.18 \\
AlexNet [22] & 57065295 & 226.63 & 87.05 \\
VGG16 [23] & 134321999 & 731.75 & 88.72 \\
SqueezeNet [24] & 743119 & 92.66 & 89.41 \\
MVF-Net (ours) & 23705055 & 384.75 & 92.27 \\
\hline
\end{tabular}

comprehensive; 3) the embedding loss introduced in this paper reduces the distance between the centers of each class and fully explores the correlation between the features, so that the learned features are more discriminative.

\section{CONCLUSIONS}

This paper proposes a multi-view fusion network (MVF-Net) for crop disease recognition. The proposed MVF-Net is composed of a suppression branch and a global branch. The suppression branch forces the network to find subtle discriminative features in confusing categories. The global branch directly inputs the original image into the network to learn the global feature of the image. In addition, we also introduce an embedding space, in which the samples are embed into a low-dimensional space, and a effective embedding loss function is constructed for training the model and capturing as many intra-class differences as possible. The recognition process of the proposed MVF-Net comprehensively utilizes global and local features, making the recognition basis more comprehensive. It can not only take care of the global feature, but also subtly capture the fine-grained features to realize accurate crop disease recognition. The experimental results show the effectiveness and superiority of the proposed MVF-Net.

\section{REFERENCES}

[1] Q. Zhang, B. Sun, Y. Cheng, and X. Li, "Residual self-calibration and self-attention aggregation network for crop disease recognition," International Journal of Environmental Research and Public Health, vol. 18, no. 16, p. 8404, 2021.

[2] J. Trivedi, Y. Shamnani, and R. Gajjar, "Plant leaf disease detection using machine learning," in International Conference on Emerging Technology Trends in Electronics Communication and Networking. Springer, 2020, pp. 267-276.

[3] T.-Y. Lin, P. Dollár, R. Girshick, K. He, B. Hariharan, and S. Belongie,"Feature pyramid networks for object detection," in Proceedings of the IEEE Conference on Computer Vision and Pattern Recognition, 2017, pp. 2117-2125.

[4] J. Redmon, S. Divvala, R. Girshick, and A. Farhadi, "You only look once: Unified, real-time object detection," in Proceedings of the IEEE Conference on Computer Vision and Pattern Recognition, 2016, pp. 779-788.

[5] Y. Yuan, H. Ning, and X. Lu, "Bio-inspired representation learning for visual attention prediction," IEEE Transactions on Cybernetics, vol. 51, no. 7, pp. 35623575, 2021. 
[6] Y. Dong, Q.-A. Fu, X. Yang, T. Pang, H. Su, Z. Xiao, and J. Zhu,"Benchmarking adversarial robustness on image classification," in Proceedings of the IEEE Conference on Computer Vision and Pattern Recognition, 2020, pp. 321-331.

[7] Lu X, Chen Y, Li X. Hierarchical recurrent neural hashing for image retrieval with hierarchical convolutional features[J]. IEEE transactions on image processing, 2017, 27(1): 106-120.

[8] Y. Kawasaki, H. Uga, S. Kagiwada, and H. Iyatomi, "Basic study of automated diagnosis of viral plant diseases using convolutional neural networks," in International Symposium on Visual Computing. Springer, 2015, pp. 638-645.

[9] P. Sanyal and S. C. Patel, "Pattern recognition method to detect two diseases in rice plants," The Imaging Science Journal, vol. 56, no. 6, pp. 319-325, 2008.

[10] M. Agarwal, S. K. Gupta, and K. Biswas, "Development of efficient cnn model for tomato crop disease identification," Sustainable Computing: Informatics and Systems, vol. 28, p. 100407, 2020.

[11] S. Sladojevic, M. Arsenovic, A. Anderla, D. Culibrk, and D. Stefanovic,"Deep neural networks based recognition of plant diseases by leaf image classification," Computational intelligence and neuroscience, vol. 2016, 2016.

[12] S. P. Mohanty, D. P. Hughes, and M. Salathé, "Using deep learning for image-based plant disease detection," Frontiers in Plant Science, vol. 7, p. 1419, 2016.

[13] M. Brahimi, K. Boukhalfa, and A. Moussaoui, "Deep learning for tomato diseases: classification and symptoms visualization," Applied Artificial Intelligence, vol 31, no. 4, pp. 299-315, 2017.

[14] J. Amara, B. Bouaziz, and A. Algergawy, "A deep learning-based approach for banana leaf diseases classification," Datenbanksysteme für Business, Technologie und Web (BTW 2017)-Workshopband, 2017.

[15] G. Nagasubramanian, R. K. Sakthivel, R. Patan, M. Sankayya, M. Daneshmand, and A. H. Gandomi, "Ensemble classification and IoT based pattern recognition for crop disease monitoring system," IEEE Internet of Things Journal, 2021.

[16] T.-Y. Lin, A. RoyChowdhury, and S. Maji, "Bilinear cnn models for fine-grained visual recognition," in Proceedings of the IEEE International Conference on Computer Vision, 2015, pp. 1449-1457.

[17] K. He, X. Zhang, S. Ren, and J. Sun, "Deep residual learning for image recognition," in Proceedings of the IEEE Conference on Computer Vision and Pattern Recognition, 2016, pp. 770-778.

[18] X. Zheng, Y. Yuan, and X. Lu, "A deep scene representation for aerial scene classification," IEEE Transactions on Geoscience and Remote Sensing, vol. 57, no. 7, pp. 4799-4809, 2019.

[19] Y. Chen, X. Lu, and X. Li, "Supervised deep hashing with a joint deep network," Pattern Recognition, vol. 105, p. 107368, 2020.

[20] F. Schroff, D. Kalenichenko, and J. Philbin, "Facenet: A unified embedding for face recognition and clustering," in Proceedings of the IEEE Conference on Computer Vision and Pattern Recognition, 2015, pp. 815-823.

[21] Y. Wen, K. Zhang, Z. Li, and Y. Qiao, "A discriminative feature learning approach for deep face recognition," in European Conference on Computer Vision. Springer, 2016, pp. 499-515.

[22] A. Krizhevsky, I. Sutskever, and G. E. Hinton, "Imagenet classification with deep convolutional neural networks," Advances in neural information processing systems, vol. 25, pp. 1097-1105, 2012.

[23] K. Simonyan and A. Zisserman, "Very deep convolutional networks for large-scale image recognition," arXiv preprint arXiv:1409.1556, 2014

[24] F. N. Iandola, S. Han, M. W. Moskewicz, K. Ashraf, W. J. Dally, and K. Keutzer, "Squeezenet: Alexnet-level accuracy with $50 \mathrm{x}$ fewer parameters and $0.5 \mathrm{mb}$ model size," arXiv preprint arXiv:1602.07360, 2016. 